\title{
ELENCO DE EJEMPLARES DE EDICIONES TEMPRANAS \\ DEL TEXTO ORIGINAL Y DE TRADUCCIONES DE LA OBRA DE FERNANDO DE ROJAS EN CANADA, ESTADOS UNIDOS Y PUERTO RICO
}

\author{
Erna Berndt-Kelley \\ Smith College
}

Sabido es que ya en el siglo dieciséis la obra maestra de Fernando de Rojas habia encontrado su lugar entre los libros solicitados por lectores en el Nuevo Mundo. Basta consultar documentos como las escrituras y los pagarés de escribanos y mercaderers de libros de ciudades coloniales españolas como México y Lima, para comprobar el interés por la obra en aquella época. Es más, si, como señaló Irving Leonard, en la Argentina, en el año 1597, el obispo Fernando de Trejo y Sanabria, convenció a los miembros de un sinodo en Tucumán a que se adoptase la resolución de mander

"a todas las personas, hombres y mujeres de todo nuestro obispado de cualquier estado y condición que sean, que so pena de excomunión"1

les trajesen y enviasen entre otras obras todos los ejemplares del "libro que se titula de Celestina," para que fuesen quemados, es lógico asumir que un buen numero de ediciones de la Tragicomedia de Calisto y Melibea circulaba entre los lectores en Hispanoamérica.

Desafortunadamente ningún ejemplar de los aludidos parece haberse conservado. Todos los esfuerzos que hemos hecho para localizar ejemplares de ediciones tempranas de la Comedia o de la Tragicomedia en Hispanoamérica, han resultado infructuosos. Ninguna de las bibliotecas que hemos podido consultar personalmente en Hispanoamérica, 0 a las que nos hemos dirigido por carta, cuentan con un solo ejemplar de una de las muchas ediciones de Celestina que se publicaron entre 1499 y 1631 en España y de las que, indudablemente, algunas fueron llevadas al Nuevo Mundo.

En cambio, valiosos ejemplares de muchas de las ediciones del texto original y de numerosas traducciones de la obra de Fernando de Rojas se conservan en los Estados Unidos. Recogidos por bibliófilos y bibliotecarios, legados por profesores a ciertas bibliotecas, o comprados 


\section{CELESTINESCA}

por las mismas, los ejemplares se hallan generalmente bien conservados en colecciones de libros raros.

Creyendo que seria de ayuda a los investigadores interesados en Celestina tener a mano la lista de los ejemplares que se pueden consultar en este pais, en Canadá y en Puerto Rico, quisiera compartir con los lectores de Celestinesca el elenco compilado dando, cuando existen, las signaturas de catálogo asignadas a los ejemplares. Para dar una visión de conjunto, además de enumerar los ejemplares cuya existencia 0 localización se desconocia o habia pasado desapercibida hasta ahora, incluyo los que se hallan en la Hispanic Society of America, descritos o citados en el libro de Clara L. Penney, ${ }^{2}$ como también los adquiridos por esa institución después de la publicación de su libro.

Por razones de uniformidad utilizo en la mayoria de los casos las siglas del National Union Catalog ${ }^{3}$ y ofrezco al final de este trabajo la aclaración de las abreviaturas de las bibliotecas donde se hallan los ejemplares y las señas correspondientes.

\section{EJEMPLARES DE EDICIONES TEMPRANAS DEL TEXTO ORIGINAL EN ESPAÑOL:}

1. [Comedia de Calisto y Melibea].

[Burgos, Fadrique de Basilea 1499].

NNH: I. Penney, pp. 28-33.

2. Tragicomedia de Cali//sto y Melibea...

Sevilla, Jacobo Cromberger. 1502 [ca. 1513-1515].

MiU: Rare Book Room PQ 6426.Al 1502?

3. Tragicomedia de Calisto y Meli//bea:...

Sevilla. 1502. [Roma, Marcellus Silber. ca. 1516].

MB: $\quad$ *XD .170B .9. Este ejemplar, originalmente de Vincenzio Zolla, cuyas inscripción y anotaciones contiene, perteneció a J.P.R. Lyell de quien lo obtuvo Philip Hofer. Luego lo adquirió H. P. Kraus de quien lo compró la Boston Public Library el 17 de octubre de 1941. Con las ocho octavas finales en el folio $U^{{ }^{V i}} r$, difiere del otro ejemplar conservado de esta edición que se halla en la British Library en Londres: C.20.b.15. en el cual, en el folio correspondiente aparece solamente una octava final y la marca del impresor. (V. ilustración, 32). 


\section{CELESTINESCA}

Ver Norton, pp. 152-53.

4. Tragicomedia de Calisto y Melibea...

"de Salamanca." 1502. [Roma, Antonio de Salamanca. ca. 1520].

NNH: II. Penney, pp. 33-40. Ver Norton, pp. 153-54.

5. Tragicomedia de Calisto y $\mathrm{Me}=/ /$ libea...

Sevilla. [Venecia, Juan Batista Pedrezano]. 1523.

NNH: III, Penney, pp. 40-42.

DLC: $\quad$ Rosenwald Collection 47-43824 PQ6426 .Al 1523.

6. Tragicomedia de Cali//sto y Melibea...

[Sevilla], "Iacobo Cromberger aleman y Juan cromberger," en fin de março" 1528.

NNH: Adquirido en 1956. En una nota en el interior de la cubierta delantera se lee: "2.8.56 Gift of A. M. Huntington," es decir, pasó a la Hispanic Society of America después de la publicación del libro de Penney.

7. Tragicomedia de Calisto y // Melibea...

Venecia, Juan Batista Pedrezano. 24 de octubre, 1531.

DFo: $\quad$ PQ6426 .A1 1531 Cage.

PrSjC: No catalogada.

8. Tragicomedia de Calisto y // Melibea...

Venecia, Estephano da Sabio. 10 de julio, 1534.

MB: $\quad$ XG .3356.12.

MH: $\quad$ *SC5 R6382C 1534.

NNH: IV, Penney, pp. 42-43.

WMU: $\quad$ Rare PQ 6426 .Al 1534. Ejemplar que pertenecio a J. Homer Herriott.

9. Tragicomedia de Calisto y // Melibea... Co[n]el tratado // de Centurio y el auto de Traso.

Toledo, Juan de Ayala. 28 de febrero, 1538.

NNH: V, Penney, pp. 44-45.

10. TRAGICO=//MEDIA DE CALISTO // Y MELIBEA...

Enueres, Guillome Montano. 28 de junio, 1539. 


\section{CELESTINESCA}

MB: $\quad{ }^{*} D .178 .24$ En una etiqueta en el interior de la cubierta delantera se lee: "from the Ticknor Fund // Recd. Nov. 11, 1902."

NNH: VI, Penney, pp. 45-46.

11. Siguese la tragi=//comedia de Calisto y Melibea: nue//uamente trobada y sacada de pro//sa en metro castellano por Jua[n] sedeño

Salamanca, Pedro de Castro, 15 de diciembre de 1540.

NNH: VII, Penney, pp. 46-48.

12. TRAGI=//COMEDIA DE CA=//listo y Melibea...

Enueres, Martin Nucio. [ca. 1544].

NNH: VIII, Penney, pp. 49-50.

13. TRAGI=//COMEDIA DE//Calisto y Melibea...

Enueres, Martin Nucio. 1545.

NNH: IX, Penney, pp. 50-51.

14. Tragicomedia de Calisto y Melibea.

Çaragoça, Diego Hernandez. 22 de abril, 1545.

MB: $\quad$ **G.3356 .13. Ejemplar que perteneció a Thomas Pennant Barton y fue legado a la Boston Public Library en mayo de 1873.

15. Tragicomedia//de Calisto y Melibea...

Çaragoça, George Coci. "A costas de Pedro ber=//nuz y Bartolome de nagera." 17 de junio, 1545.

NNH: $\quad X$, Penney, pp. 52-53.

16A. TRAGICOMEDIA // DE CALISTO Y MELIBEA,... mostrandoles $/ /$ los engaños que estan encerrados en // siruientes y alcahuetas. // CON SVMMA DILIGENTIA COR=//regida por el. s. Alonso de Vlloa; e impressa en // guisa hasta aqui nunca vista. E nueua $=/ /$ mente annadido el tractado // de Centurio, // CON VNA EXPOSITION DE MVCHOS // Vocablos Castellanos en lengua Thoscana.

Venecia, Gabriel Giolito de Ferrariis y svs hermanos. 20 de enero de 1553.

NNH: XI, Penney, pp. 53-55.

16B. TRAGICOMEDIA // DE CALISTO Y MELIBEA,... mostrandoles los $-/ /$ engaños que estan encerrados en sir=//uientes y alcahuetas.// Dirigida al illust. y muy Mag. S. el S. luan Micas, y 


\section{CELESTINESCA}

con // summa diligencia corregida por Alonso de Vlloa;// e impressa en guisa hasta aqui nunca // uista. $E$ nueuamente añadido // el tractado de Centurio, // CON VNA EXPOSITION D'ALGVNOS // Vocablos en lengua Thoscana.

Venecia, Gabriel Giolito Ferrari y svs hermanos. 1553.

JTSnow: Adquirido en 1984.

17. Tragico=//media de Calisto y me//libea...

Çaragoça, Agustin Milla[n]. 15 de mayo de 1555.

NNH: XII, Penney, pp. 56-57.

18. TRAGICOMEDIA // de calisto, Y // MELIBEA...

Venecia, Gabrel [sic] Givlito de Ferraris, y svs hermanos. 1556. [In fine:] "Acabose a xx. dias de Henero, del // año del nascimiento de nuestro // Saluador, M.D. LIII."

NNH: XIII, Penney, pp. 57-58.

NjP: $\quad$ Ex 3176.68 .324 .

19. TRAGICO//MEDIA DE CALIS=//TO Y MELIBEA:...

Barcelona, Claudi [sic] Bornat. 1561.

NNH: XIV, Penney, pp. 58-59.

20. Tragicome//dia de Calysto y Melibea...

Sevilla, Sebastian Trugillo. 1562.

NNH: XV, Penney, pp. 59-60.

21. Tragicomedia // De Calisto y Melibea...

Alcala, Francisco de Cormellas y Pedro de Robles. 1563.

"Ve[n]dese en casa de Ioa[n] dorta librero."

CU: $\quad$ Z239.1 1563 C66T7

22. TRAGICO=//MEDIA DE CALISTO//y Melibea...

Anvers, Philippo Nucio. 1568.

WU: $\quad$ Rare Book Collection 715363.

23. TRAGICOMEDIA // De Calisto y Me//libea,...

Madrid, Pierres Cosin. "A costa de Anton Garcia." 1569.

NNU-W: PQ6426.Al 1569. 


\section{CELESTINESCA}

24. Tragicomedia. // De Calisto y // Melibea:...

Salamanca, Mathias Màres. 1569.

NNH: XVI, Penney, pp. 60-62.

25. TRAGICOME // DIA DE CALISTO Y // MELIBEA...

Salamanca, Mathias Gast. "A costa de Simon Borgoñon." 1570.

MB: $\quad$ **G.3356 .14. Este ejemplar contiene al final diez páginas de notas manuscritas y en la portada se lee en nota manuscrita: "Ex libris Arblay." Perteneció a Thomas Pennant Barton de quien lo adquirió la Boston Public Library en mayo de 1873.

NNH: XVII, Penney, pp. 62-63.

26. TRAGICOMEDIA // DE CALISTO Y // Melibea...

Alcala, Iuan de Lequerica. "A costa de Iua[n] Gutierrez." 1575.

ICU: Rare Book Room PQ 6426 .Al 1575. (Ver p. 33)

27. TRAGICOMEDIA.//DE CALISTO//y Melibea...

Salamanca, Aluaro Vrsino de Portonarijs. 1575.

NNH: XVIII, Penney, pp. 64-65.

28. TRAGICOMEDIA.//DE CALI=//STO Y MELIBEA...

Sevilla, Alonso Picardo. "A costa de Pedro Miguel." 1575.

NNH: XIX, Penney, pp. 65-66.

29. TRAGICOMEDIA.//DE CALISTO Y ME//libea...

Alcala, Iuan Gracian. "A costa de Iuan Beyer." 1586.

MB: $\quad * X D \quad .170 B$.8. En el interior de la cubierta delantera de este ejemplar en una etiqueta se lee: "presented to the Public Library of the City of Boston By George Ticknor, Esq. Received Aprị 26, 1871 .

30. TRAGICOME [sic]//DE CALIS [sic]//y Melibea...

Salamanca, Iuan y Andres Renaut ....sta[sic] de Claudio Curlet lib [sic]" 1590. [Con "tassa" fechada en Madrid el 24 de enero, 1591].

NNH: $\quad X X$, Ejemplar muy mutilado. Penney, pp. 67-68.

31. CELESTINA.//TRAGICOMEDIA//DE//CALISTO Y MELIBEA... [¿Leyden?], Of icina Plantiniana, 1595. 


\section{CELESTINESCA}

CSmH: 82607.

DFo: $\quad$ PQ6426.Al 1595 Cage.

JT Snow Adquirido en 1987.

MB: $\quad$ *XD .170b .7. Ejemplar que perteneció a George Ticknor.

MWiW-C: No catalogada.

NNH: XXI, Penney, pp. 68-70.

WU: $\quad$ Rare Book Collection 903055.

32. TRAGICOMEDIA//DE CALISTO Y MELIBEA...

Tarragona, Felipe Roberto. 1595.

MH: $\quad$ *SC5 R6382C 1595.

NNH: XXII, Penney, pp. 70-71.

33. TRAGICOMEDIA//DE CALISTO Y ME=//libea...

Sevilla, Fernando de Lara. 1596.

NNH: XXIII, Penney, pp. 71-72.

34. CELESTINA .. Tragicomedia.//de//CALISTO Y MELIBEA...

[¿Leyden?] Oficina Plantiniana. 1599.

Existen por lo menos dos versiones de esta edición: 1) en la que en pág. 20, renglón 3, se lee "Celestino" (tipo ' $O$ ') y 2 ) en la que en la página correspondiente se lee "Celestina" (Tipo 'A').

ICN: $\quad$ Case Y722 .R616.

JT Snow Tipo 'O'. Adquirido en 1984.

NNH: Tres ejemplares:

1) Tipo 'A', XXIV, Penney, pp. 72-74.

2) Tipo 'O', Ejemplar No. 220 de la colección de Peeters-Fontainas (\#106 del catálogo de venta de Sotheby), adquirido en mayo de 1978.

3) Tipo 'O', adquirido, en 1986.

PPL: $\quad$ Six Rojas Log 2319.D.

PU: $\quad 868$ R63C 1599. Tipo "O."

TNVU: Special Collections S863 .23 R74c P7

35. TRAGICOMEDIA,//DE CALISTO, Y//MELIBEA,...

Madrid, Andres Sa[n]chez. "A costa de Miguel Martinez." 1601.

ICU: $\quad$ Rare Book Room PQ 6426.A1 1601.

NNH: Ejemplar adquirido en 1971.

OCH: RBR E/R. 
36. TRAGICOMEDIA//DE CALISTO Y//MELIBEA...

Çaragoça, Carlos de Lauayen y Iuan de Larumbe. 1607.

NNH: XXV, Penney, 74-76.

37. TRAGICOMEDIA//DE CALISTO//Y MELIBEA...

Madrid, Iuan de la Cuesta. "A costa de Miguel Martinez." 1619.

NNH: XXVI, Penney, pp. 76-77.

38. CELESTINA//Tragicomedia//DE//CALISTO Y MELIBEA...

Milan. "A costa de Iuan Baptista Bidelo." 1622.

MiDW: (Closed) PQ 6426 .Al 1622.

NNH: XXVII, Penney, pp. 77-79.

NSyU: Von Ranke Collection 863.23 R74.

TNVU: C1 863R63c 1622.

39. TRAGICOMEDIA//DE CALISTO,//Y MELIBEA, VVLGAR= $/ /$ mente llamada Celestina:...

Madrid. Viuda de Alo[n]so Martin. "A costa de Domingo Gonçalez." 1632. [In fine: 1631].

NNH: XXVIII, Penney, pp. 79-80.

40. TRAGICOMEDIA//DE CALISTO//Y MELIBEA, VVLGAR= //MENTE LLAMADA CELESTINA...// POR EL BACHILLER//Fernando de Rojas.// CORREGIDA Y EMENDADA// nueuamente y traduzida de Castellano//en Frances.

Rvan. 1633.

Edición bilingüe español/francesa. Ver pág. 23 para la portada de la traduccion francesa, también de 1633 .

NNH:

XXIX, Penney, pp. 80-82.

WHRoberts: El ejemplar carece de la portada correspondiente a la parte española.

41. TRAGICOMEDIA//DE CALISTO//Y MELIBEA, VVLGAR= //MENTE LLAMADA CELESTINA:... //POR EL BACHILLER// Fernando de Rojas.//CORREGIDA Y EMENDADA//nueuamente y traduzida de Castellano//en Frances.

Rvan. 1633.

Edición bilingüe español/francesa. Ver pág. 24 para la portada de la traducción francesa de 1634. 
MB: Dos ejemplares, ambos con una etiqueta en el interior de la cubierta delantera en la que se lee: "Bequeathed by George Ticknor. Recd. April 26th, 1871."

*D .170b .3. Ejemplar en buenas condiciones.

*D .170b .4. A este ejemplar le falta la portada correspondiente a la parte francesa. Varias de las páginas con el material preliminar se hallan traspuestas. Termina en la página 578 con el final del Acto XXI. Le faltan las ocho octavas finales que se hallan en el ejemplar ${ }^{*} D$ $.170 \mathrm{~b} .3$. En el v. de una página en blanco al principio del libro, escrito a mano aparece el siguiente:

"Epigrama ó Epitafio

\section{A Celestina}

Yaze en esta tierra fria

Digna de toda criança

La Vieja cuya alabança

Tantas plumas merecia.

No quiso en el cielo entrar

A gozar de las estrellas, Por no estar entre donzellas

Que no pudiesse manchar."

NNH: $\quad$ XXX, Penney, pp. 82-83.

\section{TRADUCCIONES:}
A. Italianas ${ }^{6}$

42. TRAGICOMEDIA DI CALI//STO E MELIBEA NOVAMEN//TE TRADVCTA DE // SPAGNOLO IN // ITALIANO // IDIOMA.

Roma, Eucharius Silber alias Franck. 29 de enero, 1506. 


\section{CELESTINESCA}

MH: $\quad$ *SC5 R6382C Ei5060

Ejemplar en excelentes condiciones. En el interior de la cubierta delantera en una etiqueta se lee: "From the Bequest of Mary P. C. Nash. In Memory of her husband Bennett Hubbard Nash. Instructor and Professor of Italian and Spanish 1866-1894."

43. TRAGICOMEDIA di Calisto e Me//libea de Lingua Hispana In Idioma Itali//co Traducta \& Nouamente Reuista: // e Correcta: e a piu lucida $\mathrm{Ve}[\mathrm{n}] \mathrm{ustate} / /$ Reducta per Hieronymo $\mathrm{Cla} / /$ ricio Immo//lese.

Milano, Zanotto da Castione ad Instantia de Domino Io. Iacobo \& fratelli da Legnano. 23 de junio, 1514.

CtY: $\quad$ Beinecke Library 198081

MH: $\quad$ *SC5 R6382C Ei506ob

44. TRAGICOComedia di Calisto: e Melibea de lingua // Hispana In Idioma Italico Traducta da Alphon//so Hordognez: \& Nouameute [sic] Reuista: e cor//recta per Vincentio minutiano,...

Milano, Officina Libreria Minutiana... Impensis Venerabilis Nicolai de Gorgonzola. Enero, 1515.

CtY: $\quad$ Beinecke Library 198048

MH: $\quad$ *SC5 R6382C Ei506oc. En el interior de la portada delantera, en una etiqueta se lee: "From the Bequest of Mary P. C. Nash In Memory of her husband Bennett Hubbard Nash Instructor and Professor of Italian and Spanish 1866-1894."

45. Tragicomedia di Calisto e // Melibea nouamente tra//ducta de Spagnolo in // Italia[n]o Idioma.

Venecia. [¿P. Pincius?]. 12 de abril, 1515.

MH: $\quad$ *SC5 R6382C Ei506od

46. Tragicocomedia di $\mathrm{Ca} / /$ listo e Melibea de Lingua Hispana In // Idioma Italico Traducta \& Noua//me[n]te Reuista: e Correcta: e a piu // lucida Venustate Reducta // per Hieronymo Clari//cio Immolese.

Milano, Ioanne Angelo Scinzenzeler. 16 de marzo, 1519.

DFo: $\quad$ PQ 6427 I8 1519 Cage

47. Celestina. // Tragicomedia de Calisto \& // Melibea nouamente tradocta de lingua castigliana // in italiano idioma. Aggiontoui di nouo tutto quel//lo ch[e] fin al giorno presente li ma[n]chaua. Da 


\section{CELESTINESCA}

poi // ogni altra impressione nouissimame[n]te correcta: distincta // ordenada: \& in piu co[m]moda forma reducta: adorna//da etiam $\mathrm{d}[\mathrm{e}]$ molte bellissime figure: segondo el nume//ro di soi acti: con le persone etia[m] a dicti acti conuenie[n]te: le qual cose nelle altre impressione non si troua.

[Venecia], Cesare Arrivabene. Diciembre 10, 1519.

Cty: Beinecke Library 1980 75. En el fol. CXVIII v. de este ejemplar aparece un autógrafo en tinta muy pálida: "Questa Commedia fù Recitada Dallo Sige [palabra ilegible] l'anno M. D. Cinque. Cioè Da [palabra ilegible] Cosimo Carlini, [palabra ilegible] Battista Calvi e Cesare Cianelli e la scena Rappresenta Pisa [palabra ilegible]."

IaU: $\quad$ XPQ 6427181519

MWelC: Plimpton 187

NNH: $\quad$ Ejemplar adquirido en 1986.

PHC: $\quad$ PQ 6427181519

48A. CELESTINA // TRAGICOMEDIA DE CALI=//STO ET MELIBEA NOVAMENTE // Tradocta de lingua castigliana in Italiano idio=//ma. Aggiontoui di nouo tutto quello che fin $/ /$ al giorno presente li manchaua. Dapoi // ogni altra impressione nouissimame[n]//te correcta, distincta, ordena//da, \& in piu commoda // forma reducta, // adornada, // lequal cose nelle altre impres=//sione non si troua.

Venecia, Gregorio de Gregorii, Noviembre, 1525.

La portada carece de marca del impresor. Folios numerados con números árabes $1-119$.

CtY: $\quad$ Beinecke Library 198073

IU: $\quad$ X862 R63 Oclo 1525

MH: $\quad$ *SC5 .R6382C .Ei506og

NNH: Ejemplar \#97 del catálogo de venta de la colección de Peeters-Fontainas, adquirido en mayo de 1978.

48B. CELESTINA // TRAGICOMEDIA DE CALISTO // ET MELIBEA NOVAMENTE // Tradotta de lingua Castigliana in Italiano idio//ma. Aggiontoui de nuouo tutto quello che fin $/ /$ al giorno presente li manchaua. Dapoi // ogni altra impressione nouissimamen//te corretta, distinta, ordinata. // \& in piu commoda forma // redotta, adornata // lequal cose nelle al//tre impressione non si troua.

Venecia, Gregorio de Gregorii. Noviembre, 1525. 


\section{CELESTINESCA}

Con marca del impresor en la portada. Folios numerados con números romanos I-CXIX.

MB: $\quad$ D .170B .5. En el interior de la cubierta delantera en una etiqueta se lee: "bequeathed by George Ticknor" y en el v. de una página en blanco al principio del libro aparece el siguiente autógrafo: "Given to me by Don José Madrazo, the King's Painter."

MH: $\quad$ Typ 52525750 . Ejemplar legado a la biblioteca el 3 de enero de 1939 por Philip Hofer. En la portada, a la izquierda del subtitulo, se lee: "Monastery"; a la derecha, "Cremifanensis," y debajo de la marca del impresor, "Nicolaus Seld." Encuadernado junto con un ejemplar de la Arcadia de Sannazaro, 1533 y con otro de Paris \& Viana, 27 de marzo, 1528.

NjP: $\quad$ Ex $3176.68 .324 \quad .8$.

48C. Edición que es combinación de "A" y de la de Caron (Ver 48). Folios C, D, E, N, y $P$ numerados con números romanos y los restantes, con números árabes.

NNH: \#98 del catálogo de la colección de J. PeetersFontainas, adquirido en mayo de 1978.

49. CELESTINA // TRAGICOMEDIA DE CALISTO // ET MELIBEA NOVAMENTE // Tradotta de lingua Castigliana in Italiano idioma. Aggiontoui di nuouo tutto quello che fin $/ /$ al giorno presente li manchaua. Dapoi // ogni altra impressione nouissimamaen $/ /$ te corretta. distinta, ordinata, $/ / \&$ in piu commoda forma // redotta, ador//nata // lequal cose nelle al//tre impressione non si troua.

Venecia, Francesco Caron. Noviembre, 1525.

Folios numerados con números romanos.

PU: $\quad 868$ R63C .10

50. Celestina [en tipos góticos]. TRAGICOMEDIA DE CALISTO ET ME//LIBEA NOVAMENTE TRADOTTA // De lingua Castigliana in Italiano idioma. Agiontoui di // nuouo tutto quello che fin al giorno presente li man=//chaua. Dapoi ogni altra impressione nouissima $=/ /$ mente corretta, distinta, ordinata, $\&$ in piu // co[m]moda forma redotta, adornata lequal $\mathrm{co} / / \mathrm{se}$ nelle altre i[m]pressione non si troua. 


\section{CELESTINESCA}

[Venecia], Marchio Sessa. 10 de febrero, 1531.

CaOTU: Thomas Fisher Rare Book Library B-10 381. Ejemplar imperfecto al que le faltan folios ix y xvi.

51. CELESTINA // TRAGICOMEDIA DI // CALISTO ET MELI//BEA, TRADOTTA // DE LINGVA CA//STIGLIANA // IN ITALIA//NO IDIO//MA. // Nuouamente ampliata \& corretta.

Venecia, Francesco di Alessandro Bindoni \& Mapheo Pasini. Junio, 1531.

NNH: \#99 de la colección de J. Peeters-Fontainas, adquirido en mayo de 1978.

52. Celestina [en tipos góticos]. // TRAGICOMEDIA DE CALISTO ET ME//LIBEA NVOVAMENTE TRADOTTA // De lingua Castigliana in Italiano idioma. A giontoui di // nuouo tutto quello che fin al giorno presente li manca=//ua. Dapoi ogni altra impressione nouissimamen//te corretta, distinta, ordinata, et in piu $\mathrm{co}[\mathrm{m}] / /$ moda forma redotta, adornata $l e=/ /$ qual cose nelle altre impres//sione non si troua. (Ver p. 34.)

[Venecia], Pietro de Nicolini da Sabio. Julio, 1535.

CtY: $\quad$ Beinecke Library 198074.

DFo: Order \#222243.1 adquirida en 1982 y aun no catalogada. Encuadernado junto con un ejemplar del Pornoboscodidascalvs latinvs, la traducción latina de Caspar Barthius, Frankfurt, 1624.

IU: $\quad$ $\quad$ 862 R63 Oclo 1535

MH: $\quad$ *SC5 R6382C Ei506ol

Miu: $\quad$ Rare Book Room PQ 6427.181535

NIC: $\quad$ Rare PQ 6427.191535

NNH: $\quad$ XXXIV, Penney, pp. 88-89

53. Celestina [en tipos góticos]. // TRAGICOMEDIA DE CALISTO // ET MELIBEA NVOVAMENTE // Tradotta de lingua Castigliana in Italiano idioma. // Dapoi ogni altra impressione nouissimamente // corretta, distinta, ordinata, \& in piu $\mathrm{com} / /$ moda forma ridotta. // Adornata di tutte le sue figure a ogni atto correspondenti // lequal cose nelle altre impressione non si truoua.

[Venecia], Giouann'antonio e Pietro de Nicolini da Sabio. Marzo, 1541.

CtY: $\quad$ Beinecke Library 198061

DFo: $\quad$ PQ 6427 .I8 1541 Cage

IaU: $\quad x P Q 6427.18 \quad 1541$ 


\section{CELESTINESCA}

ICU: $\quad$ Rare Book Room PQ 6427 .I806 1541

IU: $\quad$ Rare Book Room - No catalogada

MB: $\quad$ *XD .541.R63C

NjP: $\quad$ Ex 3176.68 .324 .5

NN: $\quad$ Spencer Coll. Ital. 1541 74-263

NNH: $\quad$ XXXV, Penney, pp. 90-91

PrSjC: No catalogada

PU: $\quad$ SC5 .R6328 .Ei54lc

54. CELESTINA // TRAGICOCOMEDIA DI // CALISTO ET MELIBEA // Nuouamente Tradotta de Spagnolo [sic] // in Italiano idioma.

Venecia, Bernardino de Bendoni. 1543.

CtY: $\quad$ Beinecke Library 198082

MnU: Z861 R63 JC

PU: $\quad$ SC5 .R6382 .Ei5050 1543E

\section{B. Francesas}

55. Celestine en la=//quelle est traicte des deceptions des seruiteurs // enuers leurs maistres \& macquerelles en=//uers les amoureux tra[n]slate dytalie[n] en fra[n]cois.

Paris, Nicolas Cousteau pour Galiot du Pré. 1 de agosto, 1527.

MH: $\quad$ *SC5 R6382C EL527c. Ejemplar de la tirada 'A' de la primera edición de la primera traducción francesa, que es anónima. ${ }^{7}$ (Brault, pp. 213). En excelentes condiciones.

56. CELESTINE EN LAQVEL=//le est Traicte des deceptions des serui=//teurs enuers leurs Maistres/ et // des Macquerelles enuers // les Amoureux. ... On les ven a Paris a la gran Rue // sainct Iacques deuant leglise des $\mathrm{Ma}=/ /$ thurins a lenseigne de Lelefant.

Paris, Nicolas Barbou. 1542.
MH:
*SC5 R6382 Eh 527ce
PU:
SC5 .R6382 .Eh542c

Ambos son de la tirada ' $C$ ' de la cuarta edición de la primera traducción francesa, que es anónima. (Brault, pp. 214-215).

57. LA // CELESTINE // FIDELLEMENT RE-//PVRGEE, ET MISE EN // meilleure forme par IACQVES // DE LAVARDIN, Escuyer, Sei-//gneur du Plessis Bourrot en Tou-//raine. // TRAGICOMEDIE IADIS // Espagnole, co[m]posee en reprehensio[n] des fols // amoureux,... // Vetula praua cauda Scorpionis. // Plus la Courtizane de Ioachim du Bellay. 


\section{CELESTINESCA}

Paris, Nicolas Bonfons. s.f. [¿1578?]

DFo: PQ 6427 F6 1578 Cage. A este ejemplar se le añadió a mano, al pie de la portada, la fecha 1578 , probablemente porque lleva el prefacio fechado Marzo, 1578.

MH: $\quad$ *SC5 R6382C Eh577lc. Adquirida el 10 de febrero, 1931 "From the Bequest of Mary P. C. Nash In Memory of her husband Bennett Hubbard Nash, Instructor and Professor of Italian and Spanish 1855-1894."

PU: $\quad$ SC5 R6382 Eh5601 1578

Según Brault (p. 216), tanto el ejemplar en la biblioteca de Harvard University como también el de la Universidad de Pennsylvania, proceden de la tercera edición de la segunda traducción francesa hecha por Jacques de Lavardin.

Como queda indicado en el título, cada ejemplar se halla encuadernado con otro de La Vieille Courtisane de Du Bellay, además de la "Elegie" de Chrestien.

58. LA // CELESTINE // TRAGICOMEDIE, // Traduit d'Espagnol en François. // Où se voyent les ruses \& tromperies, don // les maquerelles vsent enuers les // fols Amoureux. // DERNIERE EDITION.

Roven, Theodore Reinsart. 1598.

NNH: XXXII, Penney, pp. 85-87. Según Brault (pp. 21617), el ejemplar es uno de la cuarta edición de la segunda traducción francesa hecha por Jacques de Lavardin.

59. LA // CELESTINE // TRAGICOMEDIE, // Traduit d'Espagnol en François. // Où se voyent les ruse \& tromperies, dont // les maquerelles vsent enuers les // fols Amoureux. // DERNIER EDITION.

Roven, Clavde le Villain, 1599.

NNH: XXXIII, Penney, pp. 87-88. Según Brault (pp. 216-17) se trata de un ejemplar de la tirada ' $C$ ' de la cuarta edición de la segunda traducción francesa hecha por Jacques de Lavardin.

(40). LA CELESTINE // OV // HISTOIRE // TRAGICOMIQVE // DE CALISTE ET // DE MELIBEE. // Composée en Español [sic], par le Bachelier Fernam Rojas. // Et traduite de nouueau en François. 


\section{CELESTINESCA}

Roven, Chez Charles Osmont, 1633.

Edición bilingüe español/francesa. Ver p. 16 para la portada correspondiente al texto español, también de 1633.

NNH: XXIX, Penney, pp. 80-82. Según Brault (pp. 21718), el ejemplar es de la tirada ' $A$ ' de la tercera traducción francesa de un traductor anónimo.

WHRoberts

(41). LA CELESTINE // OV // HISTOIRE // TRAGICOMIQVE // DE CALISTE ET // DE MELIBEE. // Composée en Español [sic], par le Bachelier // Fernam Rojas. // Et traduite de nouueau en François.

Roven, Chez Charles Osmont. 1634.

Edición bilingüe español/francesa. Ver pág. 16 para la portada correspondiente al texto español, de 1633.

MB: $\quad$ Dos ejemplares: ${ }^{*} D .170 b .3$ y ${ }^{*} D .170 b \quad .4$. Ver pág. 16 y 17 para más detalles.

NNH: $\quad$ XXX, Penney, pp. 82-83.

\section{Alemana}

60. Ain Hipsche Tragedia // vo[n] zwaien liebhabend[e]n // mentschen ainem Ritter // Calixtus v[o]n ainer Edl[e]n // junckfrawen Melibea ge//na[n]t // deren anfa[n]g muesa[m] // was // das mittel siesz mit // de[m] aller bittersten jr bay//der sterben beschlossen.

Augsburg, Sigismund Grymm y Marx Wirsung. 20 de diciembre, 1520.

NNM: $\quad 31.73 .2$.

\section{Flamenca}

61. Celestina, een tragicomedie van Calisto ende Melibea... Ghetranslateert wt de Spaensche in onse Nederduytsche sprake,,, Antwerp, H. Heyndricxz. [1574].

DLC: $\quad$ Rosenwald Collection PQ 6427 D8 1574.

\section{E. Latina}

62. PORNOBOSCODIDASCALVS // LATINVS. // DE LENONVM, LE-//NARVM, CONCILIATRICVM, // SERVITIORVM, DOLIS, VENEFI-//ciis, machinis, plusquam Diabolicis, de miseriis iuvenum incautorum // qui florem aetatis Amoribus incocessis 


\section{CELESTINESCA}

addicunt; de Miserabili // singulorum periculo \& omnium interitu. // LIBER PLANS DIVINVS. // LINGVA HISPANICA AB IN//CERTO AUCTORE INSTAR LVDI // conscriptus CELESTINAE titulo, Tot vitae instruen-//dae sententijs, figuris, monitis, // plenus, vt par aliquid nulla ferè lingua // habeat. // CASPAR BARTHIUS...

Francofvrti, Typis Wechelianis apud Danielem \& Dauidem Aubrios \& Clementem Schleichium. 1624.

Al final el 'Leander' en latín de Barthius seguido de una traducción latina del poema 'Hero \& Leander' del pseudo Museus.

$\begin{array}{ll}\text { Cty: } & \text { Zg17 B28 624p } \\ \text { CU: } & \text { PQ 6427 L3B3 1624 } \\ \text { DFo: } & \text { Dos ejemplares: }\end{array}$

1) PQ 6427 L2 1624 Cage

2) 222243.1 Aun no catalogado. Encuadernado junto con un ejemplar de la traducción italiana Celestina, [Venecia], Pietro de Nicolini da

MB: D.167.21 Sabio, Julio, 1535. Ver \#52.

MH: $\quad$ *SC5 R6382C Ef624b

NjP: $\quad$ Ex 3176.68 .324 .57

NNH: XXXVI, Penney, pp. 91-93.

PU: $\quad 868 . R 63 C . L B$

TNVU: PQ6427.L2 .B3.

TSBeardsley: Ejemplar que perteneció a Peeters-Fontainas

\section{f. Inglesas}

63. THE // SPANISH BAWD // REPRESENTED // IN CELESTINA // OR, // The Tragicke-Comedy of // CALISTO and MELIBEA. // Wherein is contained, besides the pleasantness and sweetenesse // of the stile, many Philosophicall Sentences, and profitable // Instructions necessary for the younger sort: // Shewing the deceits and subtilties housed in the bosomes of false // seruants, and Cunny-catching Bawds. // [raya] // [marca del impresor] // [raya] // LONDON // Printed by J.B. And are to be sold by // ROBERT ALLOT at the Signe of the Beare // in Pauls Churchyard. 1631 .

$\begin{array}{ll}\text { CSmH } & \text { STC 4911 } 99589 \\ \text { CtY } & \text { Dos ejemplares: } \\ & \text { 1) Beinecke Library He43 +22Hm } \\ & \text { 2) Elizabethan Club } \\ \text { CU } & \text { PQ } 6427 \text { E36 1631 } \\ \text { DFo } & \text { STC } 4911\end{array}$




\section{CELESTINESCA}

$\begin{array}{ll}\text { DLC } & \text { PQ 6427.E56 1631 } \\ \text { FU } & \text { 862.2 R74lcEm Rare Books } \\ \text { ICN } & \text { fy 1565.R64 } \\ \text { ICU } & \text { PQ6427.E5 .M19 Rare Book Room } \\ \text { IU } & \text { STC 4911 - No catalogada } \\ \text { MH } & \text { fSTC 4911 } \\ \text { NNH } & \text { XXXI, Penney, pp. 83-85 } \\ \text { OU } & \text { PQ 6427* E5 1631 } \\ \text { TNVU } & \text { S863.3 R74cE M11 } \\ \text { TXU } & \text { PQ 6427.E56 1631 } \\ \text { NjP } & \text { RHT (Robert H. Taylor Collection) }\end{array}$

Esta edición de THE SPANISH BAWD, Londres, 1631 [STC 4911] fue añadida a y encuadernada con la edición de la traducción inglesa $T H E$ ROGVE de la obra de Mateo Alemán impresa por William Tvrner en Oxford, 1630 [STC 290]. Hemos podido localizar los siguientes ejemplares:

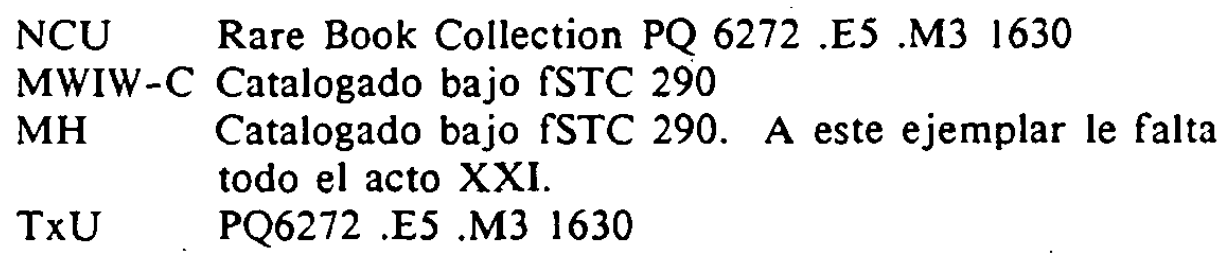

THE SPANISH BAWD, Londres, 1631 [STC 4911] tambien fue agregada a y encuadernada con ejemplares de la tercera edición de la Primera parte de THE ROGVE, impresa por Robert Allot en Londres en 1634, seguida de la Segunda parte de esa obra publicada por el mismo impresor en Londres en 1633 [STC 291]. De esta version hay ejemplares en

$\begin{array}{ll}\text { CtY } & \text { Beinecke Library He67 }+37 \mathrm{~W} \\ \text { DFo } & \text { STC Copy 1 } \\ \text { En esta biblioteca existe otro ejemplar de STC 291 } \\ \text { (Copy 2) que no contiene THE SPANISH BAWD, a } \\ \text { pesar de que en la portada se lee: "To which is } \\ \text { added, the Tragi-Comedy of CALISTO // and } \\ \text { MELIBEA, represented in Celestina. } \\ \text { Case Y 1565.A } 362 \\ \text { ICN, } \\ \text { PQ6272.E7G8 M203 } \\ \text { DH } \\ \text { 1) Keats *fEC8.K2262.R622ac } \\ \text { 2) Keats *fEC8 .K2262 Zz634a Este ejemplar } \\ \text { perteneció al poeta John Keats. Está bellamente } \\ \text { encuadernado y se guarda en una caja especial. } \\ \text { Contiene referencias sobre la procedencia y un } \\ \text { poema de James Rice dedicado a John Keats en }\end{array}$




\title{
CELESTINESCA
}

\begin{abstract}
ocasión de recibir este ejemplar de la traducción inglesa de la obra de Mateo Alemán. En una etiqueta en el interior de la cubierta delantera se

NCU lee: "Gift of // Arthur-Amory Houghton, Jr."

NN PQ 6272 .E5 .M3 1634

NNH Ejemplar cuya I Parte de THE ROGVE, como en los otros ejemplares citados, fue impresa en 1634 y cuya II Parte carece de portada y no lleva fecha de impresión. Contiene THE SPANISH BAWD.
\end{abstract}

THE SPANISH BAWD, Londres, 1631 (STC 4911) tambièn fue añadida a y encuadernada con la cuarta edición corregida de $T H E$ ROGUE "Printed by W. B. for PHILLIP CHETWIND" en Londres en 1656. De esta versión hemos podido localizar dos ejemplares:
MB $\quad{ }^{* *}$ D. 160a .76 En una etiqueta en el interior de la cubierta delantera consta: "Bequeathed by George Ticknor. Recd April 26, 1871."
NcGU Special Collection PQ 6272 .E5 M3 1656

63. THE // SPANISH BAWD // REPRESENTED // IN CELESTINA // OR // The Tragicke-Comedy of // CALISTO and MELIBEA. // Wherein is contained,... and Cunny-catching bawds. // [raya] // Marca del impresor // [raya] // LONDON // Printed by J. B[eale]. And are to be sold by // RALPH MABBE, 1631.

[STC 4911.2].

Esta edición, en la que se indica a Ralph Mabbe como vendedor, es sumamente rara. Hemos podido localizar tan sólo dos ejemplares:

$\begin{array}{ll}\text { CtY } & \text { Beinecke Library } \mathrm{He} 43+22 \mathrm{Hn} \\ \text { JTSnow } & \begin{array}{l}\text { Adquirido en 1988. Tiene ex-libris de Charles } \\ \text { Lilburn. }\end{array}\end{array}$

Un gran número de los ejemplares mencionados los he consultado personalmente. Cuando esto me ha sido imposible, me he dirigido por carta a los bibliotecarios de libros raros de las respectivas bibliotecas quienes, en la mayoria de los casos, me proporcionaron por correspondencia la información pedida, enviándome en muchos casos fotocopias de las portadas y colofones. Quisiera aprovechar esta ocasión para agradecerles su valiosa ayuda.

Por necesidad este elenco es tentativo. Sin duda habrá en las Américas otros ejemplares de estas o de otras ediciones y traducciones primitivas. Guardo la esperanza de que con el tiempo aparezcan, quizás en bibliotecas privadas, algunos de los ejemplares traidos al Nuevo Mundo por los colonizadores cuyos paraderos hoy ignoramos. 


\section{CELESTINESCA}

Mientras tanto invito y ruego a todo lector que tuviera conocimiento de ejemplares existentes en las Américas no mencionados aqui, me comunique esa información para que se pueda completar debidamente este elenco.

\section{NOTES}

${ }^{1}$ Ver Antonio E. Serrano Redonnet, "Prohibición de libros en el primer sinodo santiagueño (Tucumán)," Revista de Filología Hispánica (Buenos Aires), vol. 5., núm. 2 (1943), pp. 162-166. Citado por Irving A. Leonard, Los libros del conquistador. México: Fondo de Cultura Económica, 1959, p. 99.

${ }^{2}$ The Book Called "Celestina" in the Library of The Hispanic Society of America. New York: The Hispanic Society of America, 1954.

${ }^{3} \mathrm{He}$ examinado la gran mayoria de los ejemplares cuya localizacion se indica en The National Union Catalog Pre-1956 Imprints. Cuando no me ha sido posible consultar personalmente algunos de los ejemplares, los bibliotecarios me han confirmado la existencia--o no existencia--en sus bibliotecas de las ediciones mencionadas en dicho catálogo, cuyas páginas, desafortunadamente, contienen numerosos errores.

${ }^{4}$ Ver Frederick John Norton, "Appendix B--The Early Editions of the "Celestina'," in Printing in Spain. 1501-1520, Cambridge: The University Press, 1966, p. 151 and p. 169.

5 Penney, p. 73.

${ }^{6}$ Para una descripción más detallada de las ediciones a las que los ejemplares de las traducciones italianas mencionados a continuación pertenecen, ver Emma Scoles, "La prima traduzione italiana della Celestina: repertorio bibliográfico," Studi di Letteratura Spagnola, 1964, pp. 209-30.

${ }^{7}$ Gerard J. Brault, "Celestine": A Critical Edition of the First French Translation. (1527) of the Spanish Classic "La Celestina." with an Introduction and Notes. Detroit: Wayne State University Press, 1963. Ver especialmente "Appendix I: Editions of the Three French Translations of the 'Celestina', 1527-1644," pp. 213-18. 
Abreviaturas y señas de los lugares en el Canadá y los Estados Unidos, inclusive Puerto Rico, donde se hallan los ejemplares

CaOTU-TF Thomas Fisher Rare Book Library, University of Toronto, Toronto, Canadá M5S IA5

CSmH The Henry E. Huntington Library, 1151 Oxford Road, San Marino CA 91108

CtY The Beinecke Rare Book and Manuscript Library, Yale University, 1603-A Yale Station, New Haven CT 06520

CU The Bancroft Library, University of California, Berkeley CA 94720

DFo · The Folger Shakespeare Library, Washington, DC 20003

DLC The Library of Congress of the United States, Washington, DC 10540

FU Rare Books - Manuscripts, The University of Florida, 531 Library West, Gainesville FL 32611

IaU Special Collections, The University of Iowa Libraries, Iowa City IA 52242

ICN The Newberry Library, 60 West Walton Street, Chicago IL 60610

ICu The Joseph Regenstein Library, The University of Chicago, 1100 East 57th Street, Chicago IL 60637

IU Rare Book Room, University Library, University of Illinois at Urbana-Champaign, Urbana IL 61801

JTSnow Professor Joseph T. Snow, Department of Romance Languages, University of Georgia, Athens GA 30602

MB Department of Rare Books and Manuscripts, Boston Public Library, Boston MA 02117

MH The Houghton Library, Harvard University, Cambridge MA 02138

MiDW Special Collections, G. Flint Purdy Library, Wayne State University, Detroit MI 48202

MiU University Library, The University of Michigan, Ann Arbor MI 48109

MnU Special Collections and Rare Books, O. Meredith Wilson Library, University of Minnesota, 309 19th Avenue South, Minneapolis MN 55455 


\section{CELESTINESCA}

MWelC Special Collections, Wellesley College Library, Wellesley MA 02181

MWiW-C Chapin Library, Williams College, Williamstown MA 02167

NcGU Special Collections, Walter Clinton Jackson Library, The University of North Carolina at Greensboro, Greensboro NC 27412

NcU Rare Book Collection, Wilson Library, The University of North Carolina at Chapel Hill, Chapel Hill NC 27512

NIC Department of Rare Books, Cornell University Library, Ithaca NY 14853

NjP Princeton University Library, Princeton NJ 08544

NN The New York Public Library, Fifth Avenue at 42nd Street, New York NY 10018

NNH The Hispanic Society of America, 613 West 155th Street, New York NY 10032

NNM Metropolitan Museum of Art, Print Department, Fifth Avenue at 82nd Street, New York, NY 10028

NNU-W Elmer Holmes Bobst Library, New York University, 70 Washington Square S., New York NY 10012

NSyU The George Arents Research Library, Syracuse University, Syracuse NY 13210

$\mathrm{OCH}$ Hebrew Union College Library, 3101 Clifton Avenue, Cincinnati OH 54220

OU Rare Books and Manuscripts, University Libraries, The Ohio State University, 1858 Neil Avenue Mall, Columbus OH 43210-1286

PHC Haverford College Library, Haverford PA 19401

PPL The Library Company of Philadelphia, 1314 Locust Street, Philadelphia PA 19107

PrSjC La Casa del Libro, Calle del Cristo 255, San Juan Puerto Rico 00903

PU The Charles Patterson Van Pelt Library, University of Pennsylvania, Philadelphia PA 19104

TNVU The Jean and Alexander Heard Library, Special Collections, University Archives, Vanderbilt University, 41921 st Avenue South, Nashville TN 37240-0007. (Anteriormente TNJ: The Joint University Libraries). 


\section{CELESTINESCA}

TSBeardsley Theodore S. Beardsley, Jr., 1087 Bromley Avenue, West Englewood NJ 07666

TxU The University of Texas Library, Humanities Research Center, Austin TX 78712

WMU The Golda Meir Library, University of WisconsinMilwaukee, Milwaukee WI 53201

WHRoberts Professor William H. Roberts, 4428 Alcott Drive, Nashville TN 37215

WU Memorial Library, University of Wisconsin, 728 State Street, Madison WI 53706 


\section{CELESTINESCA}

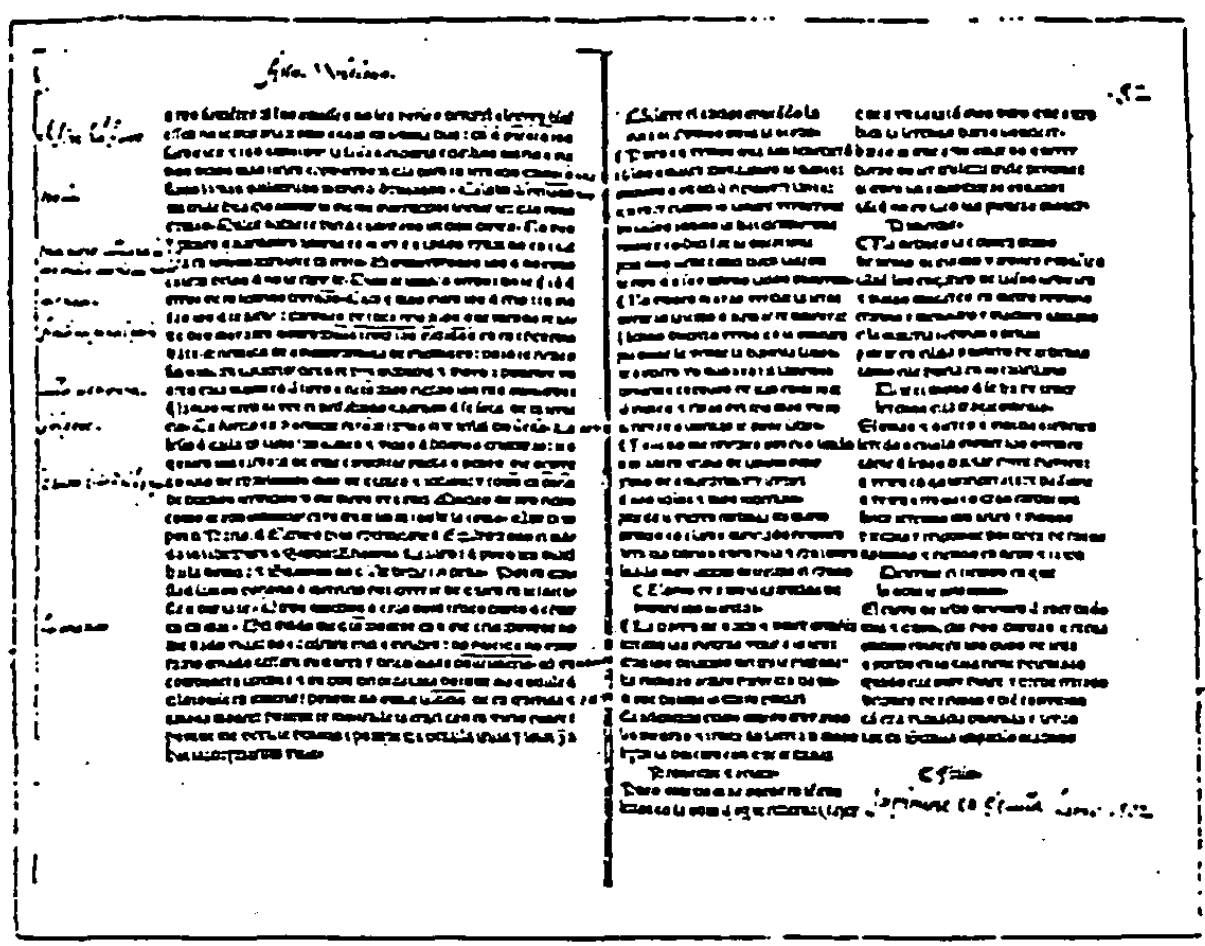

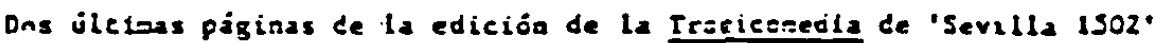
lopresa, segin F. J. Sorcon, en Rom encre 1515 y 1516 por Marcelius Silber del ejexpiar que-se conserva en la Boseon public Library (*xo .1708 .9)

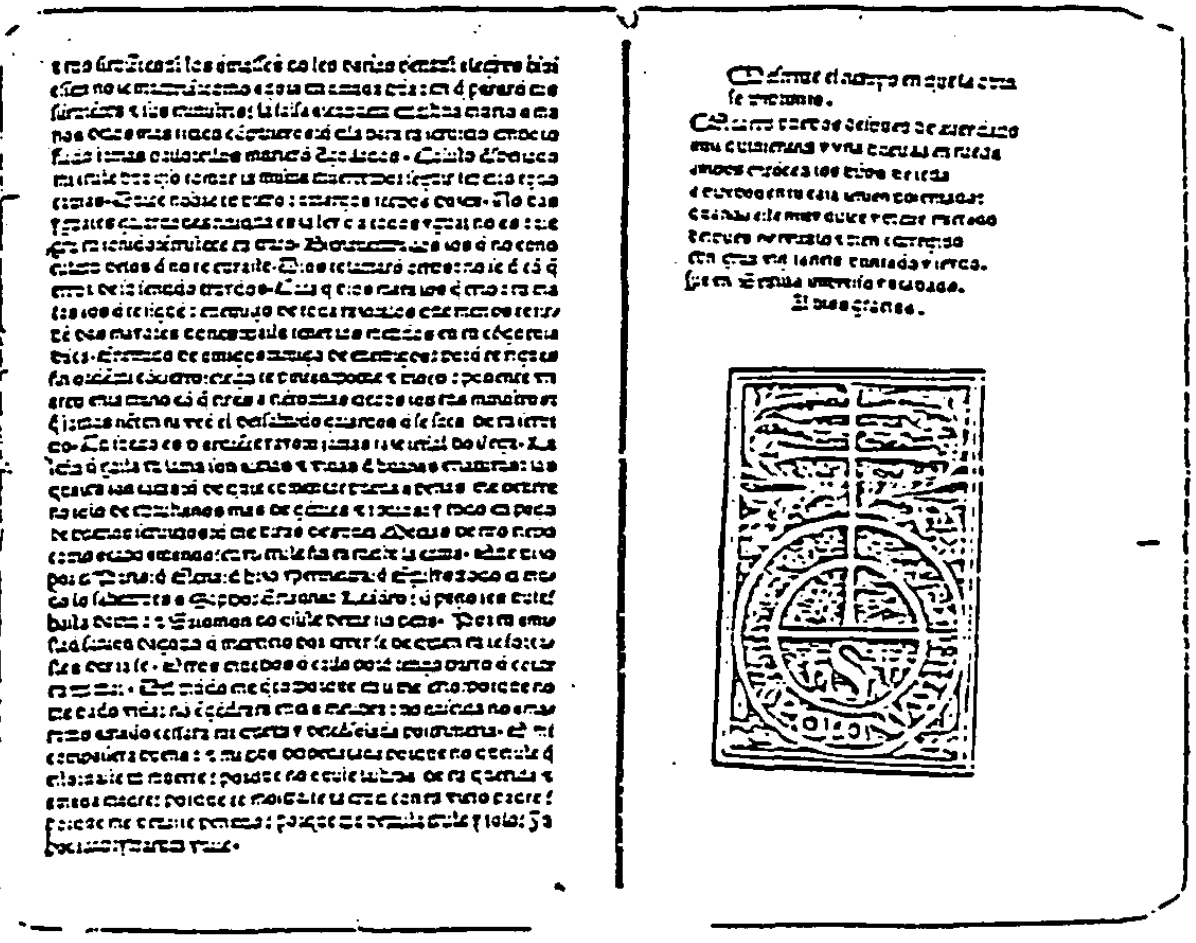

Dos úlcimas páglnas de la ediclón de la Traptcomedla de 'Sevilla 1902 ' Impresa, segun F. J. Norcon, en Rora encre 1515 y isto por Harcellus Sltber del ejemplar que se conserva en la British Library (c. 20. b. 15.) 

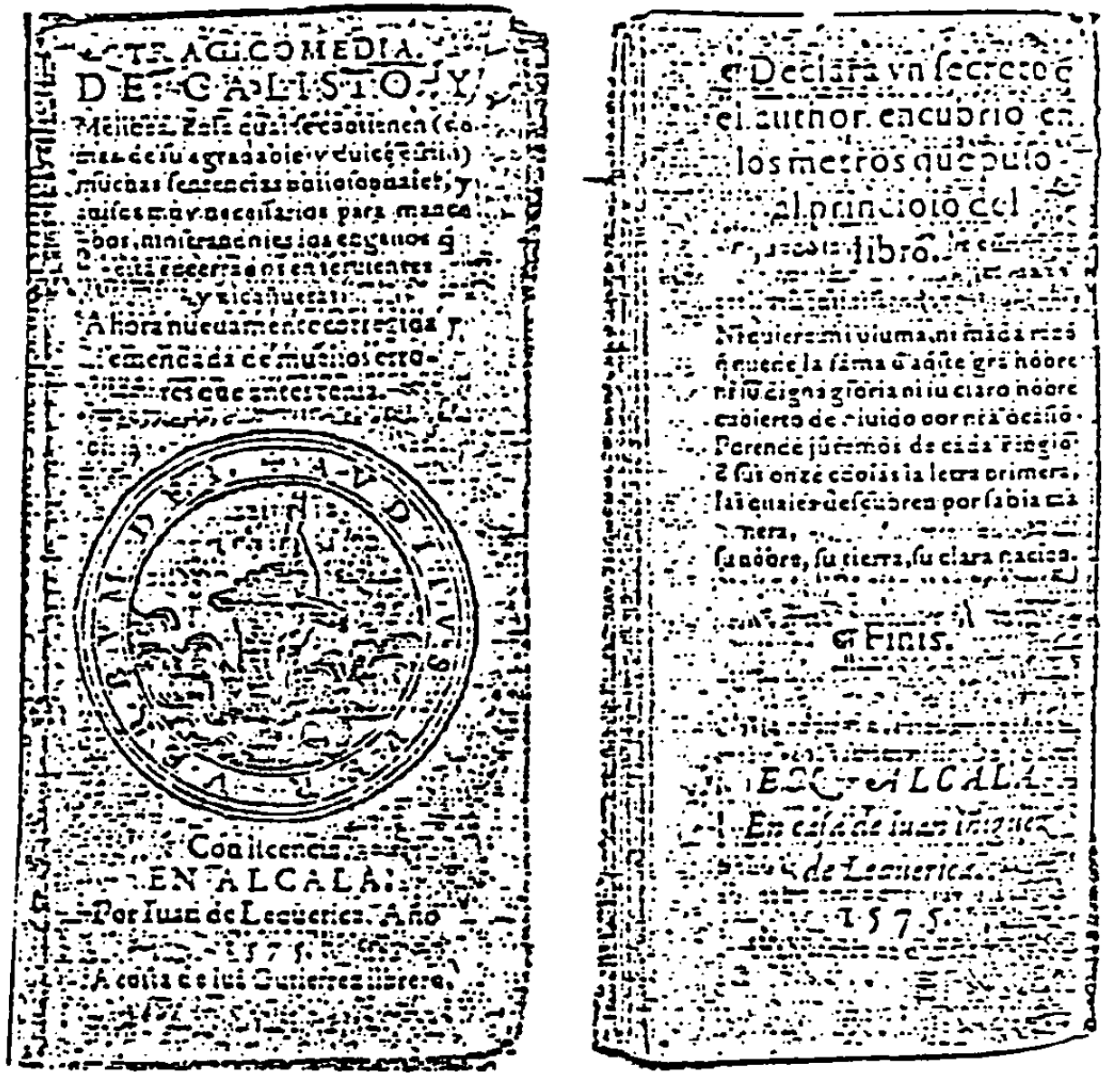

Ejemplar de la edición de Juan de Lequerica publicada en Alcala en 1575 "a costa de Iua[n] Gutierrez" que se conserva en la biblioteca de la Universidad de Chicago. 


\section{CELESTINESCA}

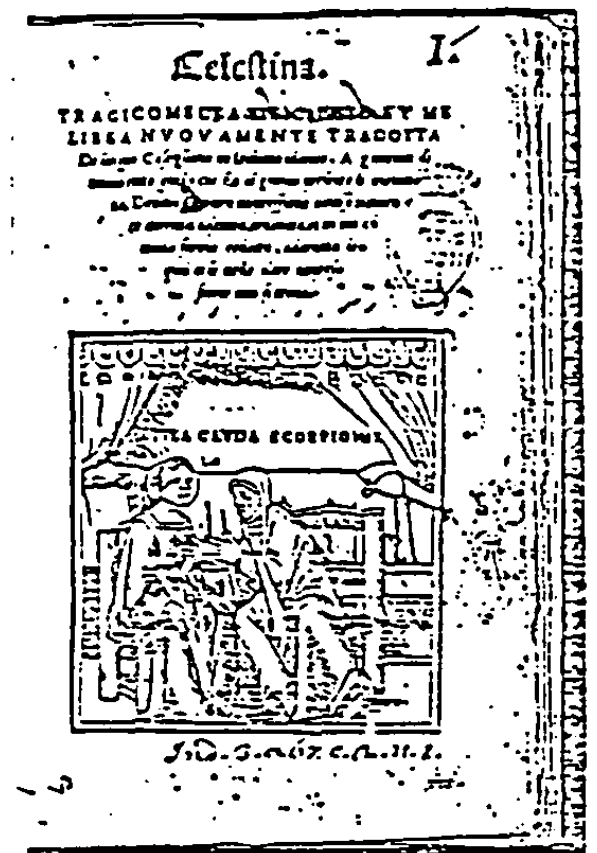

(1)

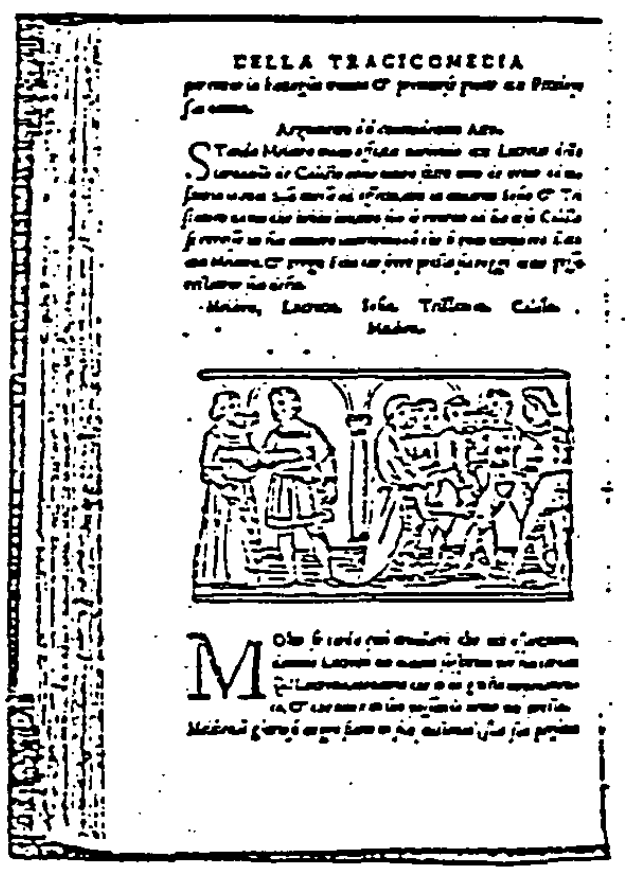

(3)

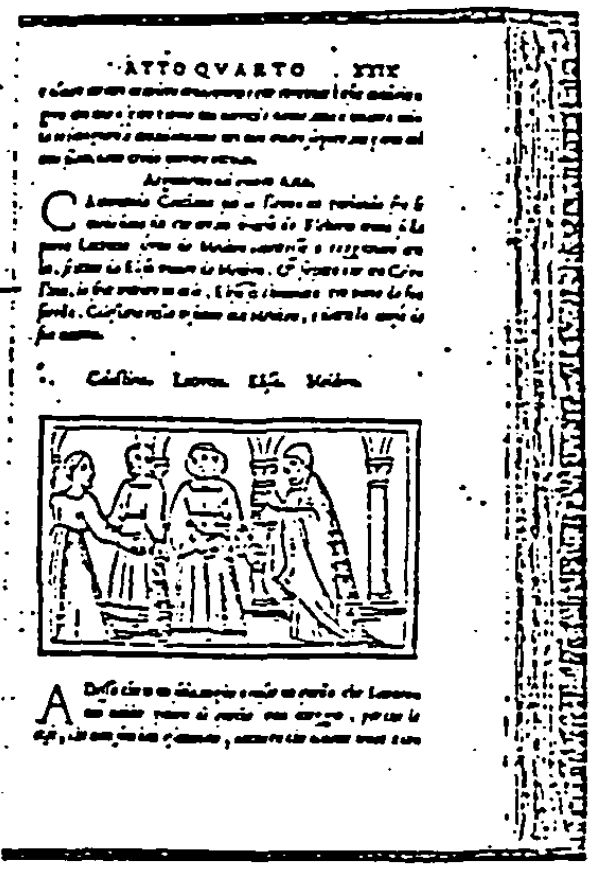

(2)

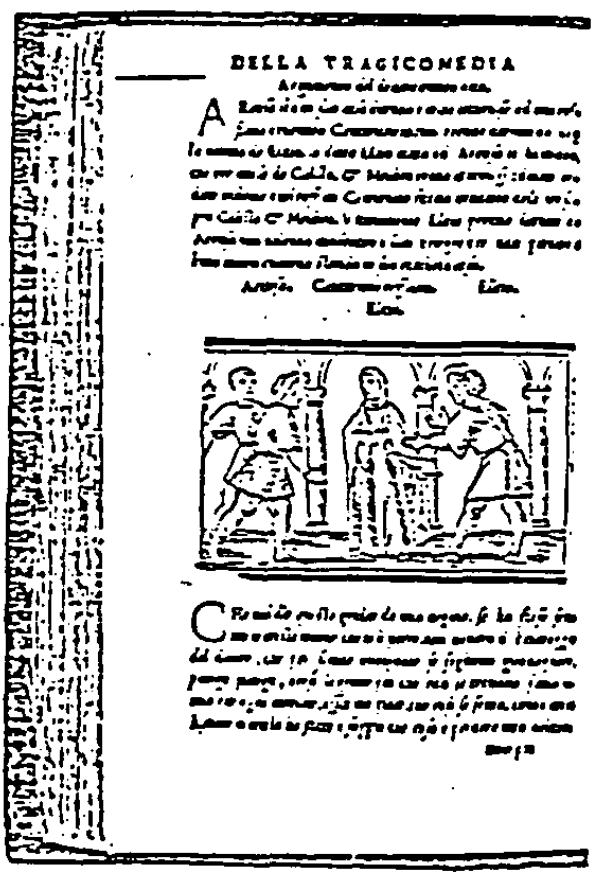

(4)

Ilustraciones en los folios I r. (1), XXIX r. (2), LXXXIII v. (3) y LXXXVIII v. (4) del ejemplar de la traducción ltaliana impresa [en Venecia] en 1535 por Pietro de Nicolini da Sabio que se halla en la Beinecke Llbrary en Yale University. Los grabados 1,3 y 4 ya habian aparecido en la edición de Cesare Arrivabene, Venecia, 1519, en la que 3 y 4 se repitieron varias veces para distincos actos. A la edición de 1535 se le añade la llustración(2) de cuarro mujeres, grabado que como(3) y (4) también se repice varias veces. 